\title{
Challenges and Opportunities
}

As the new Scientific Editor of Physiotherapy Canada, I have been examining some of the performance indicators that reflect on the journal's standing. There are countless ways to assess the successes and challenges facing a scientific publication like Physiotherapy Canada, and I will highlight a few in this editorial.

Number of submissions: Despite a below-average submission rate in the first half of 2008, Physiotherapy Canada received quite a large number of submissions in the last four months (double the rate in 2007). The editorial team was thrilled to review the 50 manuscripts submitted last year, the largest number since 2003! Building on this success, I am inviting you to continue to submit articles to the journal. You can help us meet our goal of making Physiotherapy Canada the international forum for the publication of physiotherapy-related information generated through research findings and their application in clinical practice.

Quality and types of submissions: The journal has received a variety of submissions, ranging from randomized controlled trials to case reports and systematic reviews. Although it is tempting to examine our acceptance rate as an indicator of quality, it is difficult to interpret such a statistic. For example, does a high acceptance rate reflect a high quality of submissions or a lax review process? Instead of looking at statistics only, and to ensure that we deliver a quality journal, we confirm that published articles are of the highest quality by having a distinguished editorial board with extensive academic qualifications and by relying on qualified reviewers. We also call upon other experts as needed. For example, this year a special series on pain assessment and management will be led by guest editor Dr. Judith Hunter, a physical therapist with extensive research and clinical experience in the field of pain. With the help of these experts across the country and around the world, we ensure that the journal maintains high scientific standards.

Citations and visibility: Members of CPA have free access to Physiotherapy Canada, both online and in hard copy, through our publisher, the University of Toronto Press (UTP). Moreover, articles published in the journal are available through CINAHL and AMED. To ensure that the journal continues to be widely accessible, we are actively pursuing inclusion in other databases this year.

Review process: Based on comments from authors, we have streamlined the review process. We are now using tighter timelines and new forms for providing feedback.
We have also updated the authors' guidelines, based on feedback from authors and from the editorial board, to ensure that submitted articles are reviewed more quickly. Finally, we will continue to rely on our associate editors and our more than 200 reviewers for their ongoing commitment to our journal's quality.

Reader satisfaction: A survey conducted this year about Physiotherapy Canada has revealed some interesting information. Members recognize the quality of the journal and its value both to research and to the professional identity of physiotherapy in Canada. However, members' expectations are not being fully met by the journal in its current scientific format: many readers want additional practical clinical content. Several years ago, Editor-in-Chief Susan R. Harris implemented the current system under which all articles include a Key Messages section highlighting what is already known on this subject and what this study adds. In these sections, authors summarize the relevant literature and state what their study, in particular, has added to the knowledge base. Building on this initiative, we will continue to explore and create ways to bring practical clinical content to our readers and to deliver special series on topics relevant to practice. Having said that, I believe there is also a place in Physiotherapy Canada for research that does not have direct, immediate clinical application. It is important that the journal serve as a vehicle for interchange of knowledge that not only affects practice but also generates other research questions and ideas that may eventually lead to changes in practice.

The successes of Physiotherapy Canada in the last year are due mainly to the dedication and commitment of Paul Stratford, the Scientific Editor; Susan R. Harris, the past Editor-in-Chief; and the entire editorial board. The editorial team is also indebted to the Canadian Physiotherapy Association, particularly Chris Noone, Natalie Bovair, and Michael Brennan, for their unwavering support. As we forge ahead on this path of making Physiotherapy Canada one of the best physical therapy journals in the world, we look forward to the continued support of our authors and to dialogue with you, the reader.

Dina Brooks Scientific Editor

DOI:10.3138/physio.61.2.57 